\title{
Optimal Entanglement Enhancement for Mixed States
}

\author{
Adrian Kent ${ }^{1}$, Noah Linden ${ }^{2}$ and Serge Massar ${ }^{3}$ \\ ${ }^{1}$ Department of Applied Mathematics and Theoretical Physics, University of Cambridge, \\ Silver Street, Cambridge CB3 9EW, UK \\ ${ }^{2}$ Isaac Newton Institute for Mathematical Sciences, Cambridge, CB3 0EH, UK \\ 3 Service de Physique Théorique, Université Libre de Bruxelles, CP 225, Bvd du Triomphe, B1050 Bruxelles, Belgium.
}

(4 February 1999)

\begin{abstract}
We consider the actions of protocols involving local quantum operations and classical communication (LQCC) on a single system consisting of two separated qubits. We give a complete description of the orbits of the space of states under LQCC and characterise the representatives with maximal entanglement of formation. We thus obtain a LQCC entanglement concentration protocol for a single given state (pure or mixed) of two qubits which is optimal in the sense that the protocol produces, with non-zero probability, a state of maximal possible entanglement of formation. This defines a new entanglement measure, the maximum extractable entanglement.
\end{abstract}

\section{INTRODUCTION}

Entanglement is a basic quantum communication resource which can usefully be manipulated to suit particular tasks [1,2]. In this paper we investigate the manipulation of a single entangled mixed states comprising two separated single qubit subsystems. We consider two parties, Alice and Bob, who each control one subsystem, and who are restricted to carrying out local quantum operations and classical communication (LQCC). Specifically the quantum operations Alice and Bob are allowed to perform are local unitary transformations and local filtrations. The restriction to local quantum operations ensures that entanglement is indeed treated as a resource: if non-local quantum operations were allowed, Alice and Bob could create entanglement between them from initially non-entangled states.

The interest of this problem is that since any real-world quantum communication channel will be imperfect, even if Alice could create perfect maximally entangled states, she would never be able to share such states with Bob simply by sending one subsystem through the channel. So it is natural to ask whether Alice and Bob can use LQCC to obtain states with better entanglement from imperfectly entangled states.

Various entanglement purification protocols have been suggested. If Alice and Bob share a number of copies of an imperfectly entangled known pure state, they can obtain a number of maximally entangled states by carrying out operations on each state individually or by collective operations on a number of shared states [1]. The collective algorithm has a higher asymptotic yield of maximally entangled states in the limit in which the number of shared states tends to infinity. Efficient collective algorithms which give a non-zero asymptotic yield of maximally entangled states from entangled mixed states have also been described [2].
In practice, though, the number of states will always be finite, and Alice and Bob will effectively share a single entangled state of two subsystems whose state spaces are finite dimensional. For this and other reasons - for example, Alice and Bob might actually have only one copy of an entangled state of some simple system, or it may be technologically difficult to implement collective operations - it is interesting to see what Alice and Bob can achieve by gambling with the entanglement of a single state. That is, we would like to know how far the entanglement of single states could be increased by LQCC if the outcomes of Alice and Bob's local measurements were favourable. The Procrustean algorithm of [1] provides an answer to this question in the case of pure states. Here we answer the question for two qubit mixed states, and in the process illustrate a general approach to the problem based on identifying quantities invariant under LQCC.

Though most mixed state entanglement distillation protocols discussed so far involve collective operations on many states, it has been established that there exist entangled mixed states for which single-state LQCC protocols can increase entanglement [3,4. Conversely, it is known that there exist entangled mixed states, including the important case of the Werner states, for which no single-state LQCC protocol can increase entanglement (5-7).

We give here a complete description of the effect of LQCC on entanglement of a single copy of an arbitrary mixed state $\rho$ of two qubits. It has been shown previously that if Alice and Bob's local density matrices are completely random, they cannot increase the Entanglement of Formation (EOF) by LQCC [5 77]. Here we show that if the local density matrices are not random and if the EOF is non-vanishing, Alice and Bob can always increase the EOF. Moreover we construct a procedure that maximises the EOF of the final state. This procedure, which is unique up to local unitary transformations, leaves Alice and Bob with completely random local density matrices. 


\section{MAIN RESULTS}

Throughout, the states considered are those of a single system comprising two separate single qubit subsystems. We use the following facts. [5]

1. The LQCC protocols we consider map the state $\rho$ to states of the form

$$
\rho^{\prime}=\frac{A \otimes B \rho A^{\dagger} \otimes B^{\dagger}}{\operatorname{Tr}\left(A \otimes B \rho A^{\dagger} \otimes B^{\dagger}\right)},
$$

where $A$ and $B$ are arbitrary operators that act on Alice and Bob's Hilbert space respectively. The only condition they must obey is $A^{\dagger} A \leq I_{2}$, $B^{\dagger} B \leq I_{2}$. The protocol succeeds with probability $\operatorname{Tr}\left(A \otimes B \rho A^{\dagger} \otimes B^{\dagger}\right)$. We need not consider the most general local protocols in which the final state consists of mixtures of states of the form eq. (1) since mixing decreases the EOF.

The operators $A$ and $B$ can be written as

$$
A \otimes B=U_{A} f^{\mu, a, \mathbf{m}} \otimes U_{B} f^{\nu, b, \mathbf{n}},
$$

where $U_{A}, U_{B}$ are unitary and the filtrations $f$ are defined by

$$
f^{\mu, a, \mathbf{m}}=\mu\left(I_{2}+a \mathbf{m} \cdot \sigma\right) \text { and } f^{\nu, b, \mathbf{n}}=\nu\left(I_{2}+b \mathbf{n} \cdot \sigma\right) .
$$

Here $\mu, \nu, a, b$ are real numbers, $I_{n}$ denotes the identity operator in $n$ dimensions, and the vector $\sigma=$ $\left\{\sigma_{1}, \sigma_{2}, \sigma_{3}\right\}$ has the Pauli matrices as components. We can also write these operators as $A=U_{A} F_{A} U_{A}^{\prime}$, where $F_{A}$ takes the form $\left(\begin{array}{cc}\alpha_{1} & 0 \\ 0 & \alpha_{2}\end{array}\right)$ with the $\alpha_{i}$ real, $0 \leq \alpha_{i} \leq 1$ and $U_{A}, U_{A}^{\prime}$ unitary; similarly $B=U_{B} F_{B} U_{B}^{\prime}$. We can thus write any non-trivial $\mathrm{LQCC}$ (i.e. any LQCC which is not the zero map) in the form

$$
\gamma U_{A}\left(\begin{array}{cc}
1 & 0 \\
0 & \alpha_{A}
\end{array}\right) U_{A}^{\prime} \otimes U_{B}\left(\begin{array}{cc}
1 & 0 \\
0 & \alpha_{B}
\end{array}\right) U_{B}^{\prime},
$$

where $\gamma$ is a scale factor in the range $0<\gamma \leq 1$ and $0 \leq \alpha_{A}, \alpha_{B} \leq 1$.

2. The entanglement of formation (or EOF) of a pure state $|\psi\rangle$ is defined as $E(\psi)=-\operatorname{Tr} \rho_{A} \ln \rho_{A}=$ $-\operatorname{Tr} \rho_{B} \ln \rho_{B}$ where $\rho_{A}=\operatorname{Tr}_{B}|\psi\rangle\langle\psi|, \quad \rho_{B}=$ $\operatorname{Tr}_{A}|\psi\rangle\langle\psi|$ are the local density matrices seen by Alice and Bob. For a mixed state the EOF is defined as 8]: $E(\rho)=\min \sum_{i} p_{i} E\left(\psi_{i}\right)$ where the minimum is taken over all decompositions of $\rho$ into pure states $\rho=\sum_{i} p_{i}\left|\psi_{i}\right\rangle\left\langle\psi_{i}\right|$.

In the case of a mixed state comprised of two single qubit subsystems, Wootters [10] has given an explicit formula for $E(\rho)$, verifying an earlier conjecture of Hill and Wootters [9]. Let $\tilde{\rho}=\sigma_{2} \otimes$ $\sigma_{2} \rho^{*} \sigma_{2} \otimes \sigma_{2}$. Call $\lambda_{i}$ the positive square roots of the eigenvalues of the matrix $\rho \tilde{\rho}$ written in decreasing order. Define the concurrence by

$$
C(\rho)=\max \left(0, \lambda_{1}-\lambda_{2}-\lambda_{3}-\lambda_{4}\right) .
$$

Then the EOF of $\rho$ is

$$
E(\rho)=H\left(\frac{1+\sqrt{1-C^{2}(\rho)}}{2}\right),
$$

where $H(p)=-p \log _{2} p-(1-p) \log _{2}(1-p)$.

3. Consider a general density matrix $\rho$ of two qubits. It can be written as

$$
\rho=\frac{1}{4}\left(I_{4}+\alpha . \sigma \otimes I_{2}+I_{2} \otimes \beta . \sigma+R_{i j} \sigma_{i} \otimes \sigma_{j}\right) .
$$

In [5] it was shown that under LQCC of the form eq. (2) the positive square roots of the eigenvalues of the matrix $\rho \tilde{\rho}$ transform as

$$
\lambda_{i} \rightarrow \lambda_{i}^{\prime}=\frac{\mu^{2} \nu^{2}\left(1-a^{2}\right)\left(1-b^{2}\right)}{t(\rho ; \mu, a, \mathbf{m} ; \nu, b, \mathbf{n})} \lambda_{i}
$$

where $t$ is the probability that the LQCC succeeded

$$
\begin{aligned}
& t(\rho ; \mu, a, \mathbf{m} ; \nu, b, \mathbf{n})= \\
& \mu^{2} \nu^{2}\left[\left(1+a^{2}\right)\left(1+b^{2}\right)+2 a\left(1+b^{2}\right) \mathbf{n} \cdot \alpha+\right. \\
& \left.2 b\left(1+a^{2}\right) \mathbf{m} \cdot \beta+4 a b R_{i j} n_{i} m_{j}\right]
\end{aligned}
$$

Thus the concurrence also transforms as

$$
C\left(\rho^{\prime}\right)=\frac{\mu^{2} \nu^{2}\left(1-a^{2}\right)\left(1-b^{2}\right)}{t(\rho ; \mu, a, \mathbf{m} ; \nu, b, \mathbf{n})} C(\rho) .
$$

It follows from eq. (8) that the ratios $\lambda_{i} / \lambda_{j}$ are invariant under LQCC. We add here the necessary qualification that the LQCC must be invertible.

Now our argument runs as follows. We consider states $\rho$ which have non-zero EOF and which are not Bell diagonal (recall that a state is Bell diagonal if all its eigenvectors are maximally entangled; equivalently it satisfies $\operatorname{tr}_{A}(\rho)=\operatorname{tr}_{B}(\rho)=\frac{1}{2} I_{2}$, ie. $\alpha=\beta=0$ in the expression for $\rho$ given in eq. (7)). We show in Theorem 1 that there is an LQCC protocol which increases the EOF of $\rho$ with non-zero probability. We show further in Theorem 3 that this process can be iterated to obtain an LQCC protocol which, with non-zero probability, maps $\rho$ to a Bell diagonal state with maximal EOF. In Theorem 4 we 
show that this is the unique optimal protocol up to local unitary rotations.

Theorem $1 \quad$ Let $\rho$ be a density matrix of a state with non-zero EOF written as in eq. (7). If $\alpha$ or $\beta$ are non-zero, then there is an invertible LQCC $A \otimes B$ mapping $\rho$ with non-zero probability to a density matrix $\rho^{\prime}$ with higher EOF than $\rho$.

Proof For small $a$ and $b$, eq. (10) takes the form

$$
C\left(\rho^{\prime}\right) \simeq \frac{1}{1+2 a \mathbf{m} \cdot \alpha+2 b \mathbf{n} \beta} C(\rho) .
$$

Hence if $\alpha$ or $\beta$ are non-zero and if $C(\rho)$ is non-zero we can always find an LQCC which, with non-zero probability, increases the EOF, by choosing appropriately small $a$ and $b$ and suitable $\mathbf{m}$ and $\mathbf{n}$.

We now need a technical lemma about the topology of the space $R$ of LQCC operations which do not decrease the EOF of a given $\rho$. The result, namely that $R$ is compact, is needed in Theorem 3 .

Lemma 2 Let $\rho$ have non-zero EOF. There exists a positive bound $\delta(\rho)$ such that if the state $\rho^{\prime}$ has greater EOF than $\rho$ and can be obtained from $\rho$ with non-zero probability by LQCC, then there exists some LQCC from which $\rho^{\prime}$ can be obtained from $\rho$ with probability greater than $\delta(\rho)$. Furthermore let $R$ be the space of LQCC which succeed with non-zero probability in producing a density matrix with EOF greater than or equal to that of $\rho$. Then $R$ is compact.

Proof Fix $\rho$. If we write $A \otimes B$ in the form (舟), $\rho^{\prime}$ is independent of the scale factor $\gamma$, so that any $\rho^{\prime}$ obtainable from $\rho$ can be obtained by a normalised LQCC, taking the form (4) with $\gamma=1$. For $\epsilon>0$, define $S_{\epsilon}$ to be the set of normalised LQCC of the form (A) with $\min \left\{\alpha_{A}, \alpha_{B}\right\}=\epsilon$. Let $E_{\epsilon}$ be the maximum EOF of any density matrix $\rho^{\prime}$ obtained from $\rho$ by the action (11) for some $A \otimes B$ in $S_{\epsilon}$. Since $E_{\epsilon}$ is continuous in $\epsilon$ and tends to zero as $\epsilon$ tends to zero, there is some positive $\epsilon_{0}$ such that $E_{\epsilon}$ is less than or equal to the EOF of $\rho$ for $\epsilon \leq \epsilon_{0}$ and such that $\epsilon_{0}(\rho)$ is maximal with this property. Let $T_{\epsilon_{0}}$ be the union for $1 \geq \epsilon \geq \epsilon_{0}$ of $S_{\epsilon}$. Now if a non-trivial LQCC $A \otimes B$ annihilates $\rho$, i.e. $A \otimes B \rho A^{\dagger} \otimes B^{\dagger}=0$, then $A \otimes B\left|\psi_{i}\right\rangle=0$ for all $i$ (where $\left|\psi_{i}\right\rangle$ are the eigenvectors of $\rho$ with non-zero eigenvalue). Thus either $A$ or $B$ must be a rank one projector up to a scale factor. Hence no LQCC in $T_{\epsilon_{0}}$ can annihilate $\rho$. Also $T_{\epsilon_{0}}$ is compact. So the probability $\operatorname{Tr}\left(A \otimes B \rho A^{\dagger} \otimes B^{\dagger}\right)$ of obtaining $\rho^{\prime}$ from $\rho$ via the LQCC is non-zero everywhere in $T_{\epsilon_{0}}$ and attains a non-zero lower bound $\delta(\rho)$ on the set. This is a lower bound for all LQCC increasing the $\mathrm{EOF}$ of $\rho$, since no LQCC outside $T_{\epsilon_{0}}$ does. The compactness of $R$ follows since it is a closed subset of $T_{\epsilon_{0}}$.

Theorem 3 Let $\rho$ written as in eq. (7) be a density matrix with non-zero EOF. If $\alpha$ or $\beta$ are non-zero, then there exists an invertible LQCC which, with nonzero probability, maps $\rho$ to a Bell diagonal density matrix $\rho^{\prime}$ which has the maximum EOF of any density matrix obtainable from $\rho$ by LQCC.

Proof Since by Lemma 2 the space of normalised LQCC which do not decrease the EOF of $\rho$ is compact, and the $\mathrm{EOF}$ is a continuous function, the lowest upper bound on the attainable EOF is attained by some LQCC. The corresponding density matrix $\rho^{\prime}$ must have $\alpha^{\prime}=\beta^{\prime}=0$, otherwise, by Theorem 1 , its EOF could be increased.

Theorem $4 \quad$ Let $\rho$ be the density matrix of a state with non-zero EOF. Then the Bell diagonal state $\rho^{\prime}$ which can be obtained from $\rho$ by LQCC is unique up to local unitary transformations. This $\rho^{\prime}$ has maximal possible EOF.

Proof We start by calculating the positive square roots $\lambda_{i}$ of the eigenvalues of the matrix $\rho \tilde{\rho}$. We order them as $\lambda_{1} \geq \lambda_{2} \geq \lambda_{3} \geq \lambda_{4}$. The ratios $\frac{\lambda_{i}}{\lambda_{j}}$ are invariant under the actions of invertible LQCC, see eq. (8). We characterise these ratios by the three numbers $c_{i}=\lambda_{i} / \lambda_{1}$, $i=2,3,4$.

From Theorem 3 we know that $\rho$ can be brought to Bell diagonal form by LQCC. We shall now show that the Bell diagonal form is uniquely specified, up to local unitary transformations, by the ratios $c_{i}$. To this end consider a Bell diagonal state $\rho_{R}=\frac{1}{4}\left(I_{4}+R_{i j} \sigma_{i} \otimes \sigma_{j}\right)$ with positive EOF. Local unitary operations $U_{A} \otimes U_{B}$ transform $\rho_{R}$ to $\rho_{R^{\prime}}=\frac{1}{4}\left(I_{4}+R_{i j}^{\prime} \sigma_{i} \otimes \sigma_{j}\right)$, where $R^{\prime}=\left(O_{1}\right)^{T} R\left(O_{2}\right)$ for some elements $O_{1}$ and $O_{2}$ of $\mathrm{SO}(3)$ : any pair of $O_{i}$ can be produced by suitable choices of $U_{A}, U_{B}$. By using a singular value decomposition [11] of $R$, we can find orthogonal $O_{i}$ such that $R^{\prime}$ is diagonal, so we can find local unitary operations mapping $\rho_{R}$ to the form

$$
\rho_{r_{1}, r_{2}, r_{3}}=\frac{1}{4}\left(I_{4}+\sum_{i=1}^{3} r_{i} \sigma_{i} \otimes \sigma_{i}\right),
$$

with all $r_{i}$ having the same sign and with $r_{1} \leq r_{2} \leq r_{3}$.

Now $\rho_{r_{1}, r_{2}, r_{3}}=\tilde{\rho}_{r_{1}, r_{2}, r_{3}}$, hence the eigenvalues of $\rho_{r_{1}, r_{2}, r_{3}}$ are equal to the $\lambda_{i}$. These eigenvalues are $\frac{1}{4}\left(1-r_{1}-r_{2}-r_{3}\right), \frac{1}{4}\left(1+r_{1}+r_{2}-r_{3}\right), \frac{1}{4}\left(1+r_{2}+r_{3}-\right.$ $\left.r_{1}\right), \frac{1}{4}\left(1+r_{3}+r_{1}-r_{2}\right)$. Since $\rho_{r_{1}, r_{2}, r_{3}}$ is assumed to be entangled, the $r_{i}$ are all less then or equal to zero. (This may be verified by checking that when the $r_{i}$ are all positive the concurrence vanishes). We can now express the ratio's $c_{i}$ in terms of the $r_{i}$. For instance $c_{2}=\left(1+r_{2}+r_{3}-r_{1}\right) /\left(1-r_{1}-r_{2}-r_{3}\right)$. It is straightforward to verify that the $r_{i}$ can be uniquely expressed in terms of the $c_{i}$ by inverting these equations. Therefore the Bell diagonal state of the form eq.(12) to which $\rho$ can be brought is unique. 


\section{CONCLUSIONS}

We have shown that any entangled state $\rho$ of two qubits whose local density matrices are not completely random can be brought by LQCC to a unique (up to local unitary transformations) Bell diagonal state. No other LQCC can bring $\rho$ to a state with more entanglement. To obtain an explicit expression for this optimal protocol, one should write explicitly the conditions that the density matrix $\rho^{\prime}$ obtained from has completely random local density matrices $\operatorname{Tr}_{A} \rho^{\prime}=\operatorname{Tr}_{B} \rho^{\prime}=I_{2}$. We have shown that these equations have a unique solution for the coefficients $a, \mathbf{m}, b, \mathbf{n}$ of the filtrations $f^{\mu, a, \mathbf{m}}, f^{\nu, b, \mathbf{n}}$ in eqs. (2, 3).

Our optimal protocol should be compared to the Procrustean algorithm for concentrating pure state entanglement of [1] which brings a non maximally entangled pure state to a maximally entangled pure state by LQCC. The main difference between the two methods is that the optimal mixed state protocol generally requires Alice and Bob to carry out different filtrations and then tell each other whether the filtrations have succeeded. Only if both succeed do they obtain (and know that they have) the state with maximum extractable entanglement. The Procrustean algorithm on the other hand can be realised without classical communication between Alice and Bob, or with only Alice carrying out the filtration and communicating the result to Bob.

In [5] it was noted that the ratios $c_{i}=\lambda_{i} / \lambda_{1}$ are invariant under invertible LQCC. The argument used in proving Theorem 4 also shows that for entangled states they consitute an exhaustive set. Indeed we can bring any entangled $\rho$ to the form eq. (12) which is characterised by three parameters $r_{i}$ and they are in one to one correspondence with the $c_{i}$. This gives a characterisation of locally equivalent entangled density matrices.
Our method also introduces an interesting combination of these invariants: the maximal extractable entanglement of a density matrix. This quantity provides a new characterisation of the entanglement of a state. It has the important property that it decreases under mixing (this follows from the convexity of the EOF [8]).

Acknowledgments We are very grateful to Sandu Popescu for several helpful discussions. Part of this work was caried out at the 1998 Elsag-Bailey - I.S.I. Foundation research meeting on quantum computation and at the 1998 workshop of the Benasque Center for Physics. AK thanks the Royal Society for financial support. SM is a chercheur qualifié du FNRS.

[1] C. H. Bennett, H. Bernstein, S. Popescu and B. Schumacher, Phys. Rev. A 53 (1996) 2046

[2] C. H. Bennett, G. Brassard, S. Popescu, B. Schumacher, J. A. Smolin and W. K. Wootters, Phys. Rev. Lett. 76 (1996) 722

[3] N. Gisin, Phys. Lett. A 210 (1996) 151

[4] M. Horodecki, P. Horodecki and R. Horodecki, Phys. Rev. Lett. 78 (1997) 574

[5] N. Linden, S. Massar and S. Popescu, Phys. Rev. Lett. 81 (1998) 3279.

[6] A. Kent, Phys. . Rev. Lett. 81 (1998) 2839.

[7] M. Horodecki, P. Horodecki and R. Horodecki, General teleportation channel, singlet fraction and quasidistillation, quant-ph/9807091

[8] C. H. Bennett, D. P. DiVincenzo, J. A. Smolin and W. K. Wootters, Phys. Rev. A 54 (1996) 3824

[9] S. Hill and W. Wootters, Phys. Rev. Lett. 78 (1997) 5022.

[10] W. Wootters, Phys. Rev. Lett. 80 (1998) 2245.

[11] See e.g. D.W. Lewis, Matrix Theory, World Scientific, Singapore (1991), p.262. 